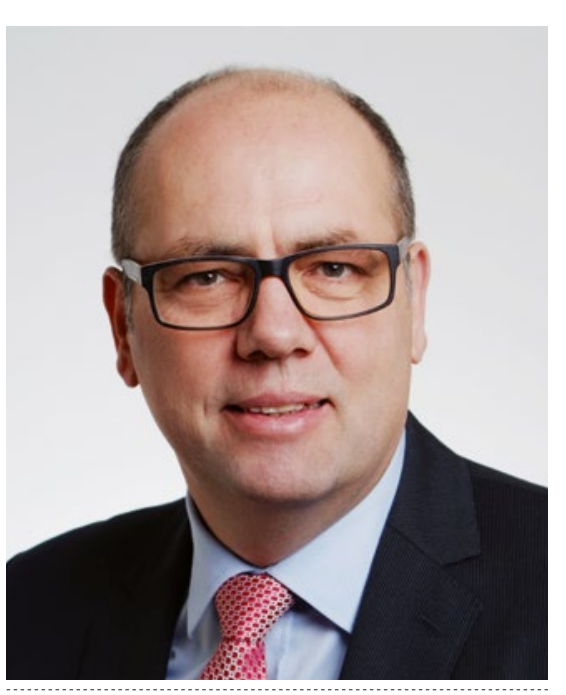

Ralph Lauxmann

ist Senior Vice President Systems \& Technology in der Division Chassis \& Safety bei Continental in Frankfurt /Main.

\title{
'||||||||||||||||||||||||||||||||||||||||||||||||||||||||||. „Wichtig ist, die kundenwerten Funktionen so schnell und gut wie möglich umzusetzen“
}

ATZelektronik_ Herr Lauxmann, so deutlich wie kein anderer Zulieferer hat sich Continental die Entwicklung von Assistenzsystemen für das hochautomatisierte Fahren auf die Fahne geschrieben. Mittlerweile dämpfen selbst OEMs wie Daimler und VW die von ihnen selbst geschürten Erwartungen. Sie stellen zumindest den Zeitplan infrage. Verunsichert Sie das nun? LAUXMANN _ Nein, auch wenn man mit überzogenen Erwartungen vorsichtig umgehen muss. Doch die Ziele in dem Rahmen, der ja gemeinsam in der Branche gesteckt wurde, lassen sich erreichen. Ich glaube, man muss die verschiedenen Begrifflichkeiten nachschärfen. Denn es gibt keine eindeutigen Definitionen, was die unterschiedlichen Ausbaustufen beziehungsweise Levels bedeuten und wie man diese auslegen kann. Die Grenzen der Levels müssen klarer gesteckt werden und vor allem, welche Funktionen jeweils darunter gefasst sind und was die Funktionen können müssen. Wir werden wahrscheinlich mit einem gewissen Mix der Levels leben müssen.

Es fehlt ein Detailierungsplan ...

... den wir derzeit erarbeiten und in die Diskussion mit einbringen werden.

Ist eigentlich klar, ob der Kunde überhaupt hochautomatisiert Fahren möchte, abgesehen von den Hürden in der Legislative?

Wichtig ist doch nur, dass wir die Inhalte und Funktionen, die wirklich kundenwert sind, so schnell und gut wie möglich - nach allen Regeln der funktionalen Sicherheit - umsetzen. Die Hochautomatisierung ist ein Ziel, doch die Funktionen, die wir im Sinne und auf ausdrücklichen Wunsch der Kunden auf diesem Weg in den Markt bringen, sind das Entscheidende. Hier lohnt jede Investition.

Die erwähnte funktionale Sicherheit für komplexe Fahrerassistenzfunktionen steht jedenfalls noch infrage, weil auch die entsprechenden Testmethoden fehlen und erst noch Standards dafür erarbeitet werden müssen. Wie beurteilen Sie das? Unsere Arbeitspakete sind zugegebenermaßen schon mächtig groß. Doch den- ken Sie an die erfolgreiche Markteinführung von Airbags und ABS- oder ESCSystemen; die Entwickler werden auch künftige Herausforderungen meistern. Dabei können wir auch auf bestehenden Standards und Baukästen aufbauen.

Mit den zahlreichen Themen der Vernetzung des Fahrzeugs kommen auch neue Zulieferer in die Prozesskette, unter anderem Unternehmen aus der Consumer-Industrie, die diese Standards und strengen Spezifikationen noch nicht kennen. Kann dies ein Sicherheitsproblem bedeuten? Ich kann mir beim besten Willen nicht vorstellen, dass ein Automobilhersteller ein Risiko eingehen würde. Es gelten ausnahmslos die gleichen Bedingungen und Anforderungen für jedes Unternehmen in der Prozesskette.

Risiken und ungelöste Aufgaben bestehen auf jeden Fall im Daten-Security-Bereich. Wie lösen Sie das mit Ihrem Partner Cisco? Wir behandeln das Thema Security mit oberster Priorität und die Sicherheitslösungen erarbeiten wir mit unseren Partnern, aber auch gemeinsam mit unseren Kunden. Gegen mögliche Angriffe müssen Fahrzeuge immer wieder von neuem gewappnet werden, weil sich Hacker immer neue Attacken ausdenken. Wir orientieren uns an den SicherheitsLeveln, die derzeit ja noch in der Abstimmung sind und die es ebenfalls immer wieder anzupassen gilt. In den entsprechenden VDA-Arbeitsgruppen sind wir aktiv involviert. Ebenso arbeiten wir an der ISO 15408 mit, der Norm zu securityrelevanten Tests, die es mit der ISO 26262 zu synchronisieren gilt.

Könnten Cracker, also die böswilligen Hacker, Ihren Zeitplan durcheinander bringen? Das glaube ich nicht.

Herr Lauxmann, ich bedanke mich für das Gespräch.

INTERVIEW: Markus Schöttle FOTо: Continental 